\title{
High Performance Liquid Chromatographic Determination of Ribonucleotides by Postcolumn Derivatization Involving Oxidation Followed by Fluorescence Reaction, and Its Application to Human Erythrocyte Sample
}

\author{
Yoshihiko Umegae, Hitoshi Nohta and Yosuke OHKURA \\ Faculty of Pharmaceutical Sciences, Kyushu University 62, Maidashi, Fukuoka 812, Japan
}

\begin{abstract}
A selective method for the determination of ribonucleotides is described which employs high performance liquid chromatography with on-line postcolumn fluorescence derivatization. Ribonucleotides are separated on a reversedphased column (TSK gel ODS-80TM) and automatically converted into fluorescent derivatives by reaction with 1,2-bis(4-methoxyphenyl)ethylenediamine in an acidic medium after periodate oxidation. The detection limits $(S / N=3)$ for ribonucleotides are $14-67 \mathrm{pmol}$ in a $100 \mu \mathrm{l}$ injection volume. The quantification of ribonucleotides in human erythrocytes is also described.
\end{abstract}

Keywords Ribonucleotide, high performance liquid chromatography, postcolumn fluorescence derivatization, 1,2bis(4-methoxyphenyl)ethylenediamine, periodate oxidation, erythrocyte

The concentrations of ribonucleotides, such as adenosine-5'-triphosphate (ATP) and adenosine-5'-diphosphate (ADP), not only reflect the activity conditions of cells and organs ${ }^{1-3}$, but also involve the degree of freshness of fish and meat food products. ${ }^{4}$

The methods for the determination of ribonucleotides in biological materials are mostly based on high performance liquid chromatography (HPLC) with UV detection..$^{5-7}$ However, these methods are neither very sensitive nor selective; very little attempt has been made to improve them regarding sensitivity and selectivity..$^{8,9}$

We recently reported that 1,2-bis(4-methoxyphenyl)ethylenediamine ( $p$-MOED) reacted selectively and sensitively with ribonucleosides and ribonucleotides in an acidic medium after periodate oxidation to produce fluorescent derivatives. ${ }^{10}$ A simple, selective HPLC method was then established for the determination of pseudouridine (a modified nucleoside as a tumor marker) in human urine and serum by applying the above-mentioned fluorescence reaction to postcolumn derivatization. ${ }^{11}$ This study extended the application of the fluorescence derivatization reaction to the HPLC determination of ribonucleotides. Ribonucleotides are separated on a reversed-phase column and are then subjected to fluorescence derivatization. Ribonucleotides in human erythrocytes were also determined by an established method using xanthine-5'-monophosphate (XMP) as an internal standard.

\section{Experimental}

\section{Reagents and solutions}

$p$-MOED was synthesized as described previously. ${ }^{12}$ All chemicals were of reagent grade. Deionized and distilled water was used. A p-MOED solution $(20 \mathrm{mM})$ and a sodium periodate solution $(2 \mathrm{mM})$ were prepared in $0.14 \mathrm{M}$ perchloric acid containing $68 \%(\mathrm{v} / \mathrm{v})$ ethanol and in water, respectively.

\section{HPLC system and its operation}

Figure 1 is a schematic diagram of an HPLC system constructed for the determination of ribonucleotides. A $100 \mu \mathrm{l}$ aliquot of sample solution was applied into a CCPM chromatograph (Tosoh, Tokyo, Japan) equipped with a Rheodyne 7125 syringe loading sample injector valve $(100 \mu \mathrm{l}$ loop) and a TSK gel ODS-80 $\mathrm{TM}$ column (particle size $5 \mu \mathrm{m}, 250 \times 4.6 \mathrm{~mm}$ i.d.; Tosoh). The mobile phase $(10 \mathrm{mM}$ sodium phosphate buffer $(\mathrm{pH}$ 4.0) containing $2 \%(\mathrm{v} / \mathrm{v})$ methanol and $5.4 \mathrm{mM}$ EDTA$2 \mathrm{Na}$ ) was delivered at a flow rate of $0.5 \mathrm{ml} \mathrm{min}^{-1}$. The column temperature was ambient $\left(25 \pm 2^{\circ} \mathrm{C}\right)$. The sodium periodate solution was added to the eluate stream by means of an SSP PM 1024 pump (Sanuki Kogyo, Tokyo, Japan) at a flow rate of $0.25 \mathrm{ml} \mathrm{min}^{-1}$; the mixture was passed through a reaction coil ( $1 \mathrm{~m} \times 0.5 \mathrm{~mm}$ i.d., stainless-steel tube) for oxidation. The $p$-MOED solution was added to the stream at a flow rate of $0.25 \mathrm{ml} \mathrm{min}^{-1}$. The mixture was then heated in a reaction coil $(20 \mathrm{~m} \times 0.5 \mathrm{~mm}$ i.d., stainless- 


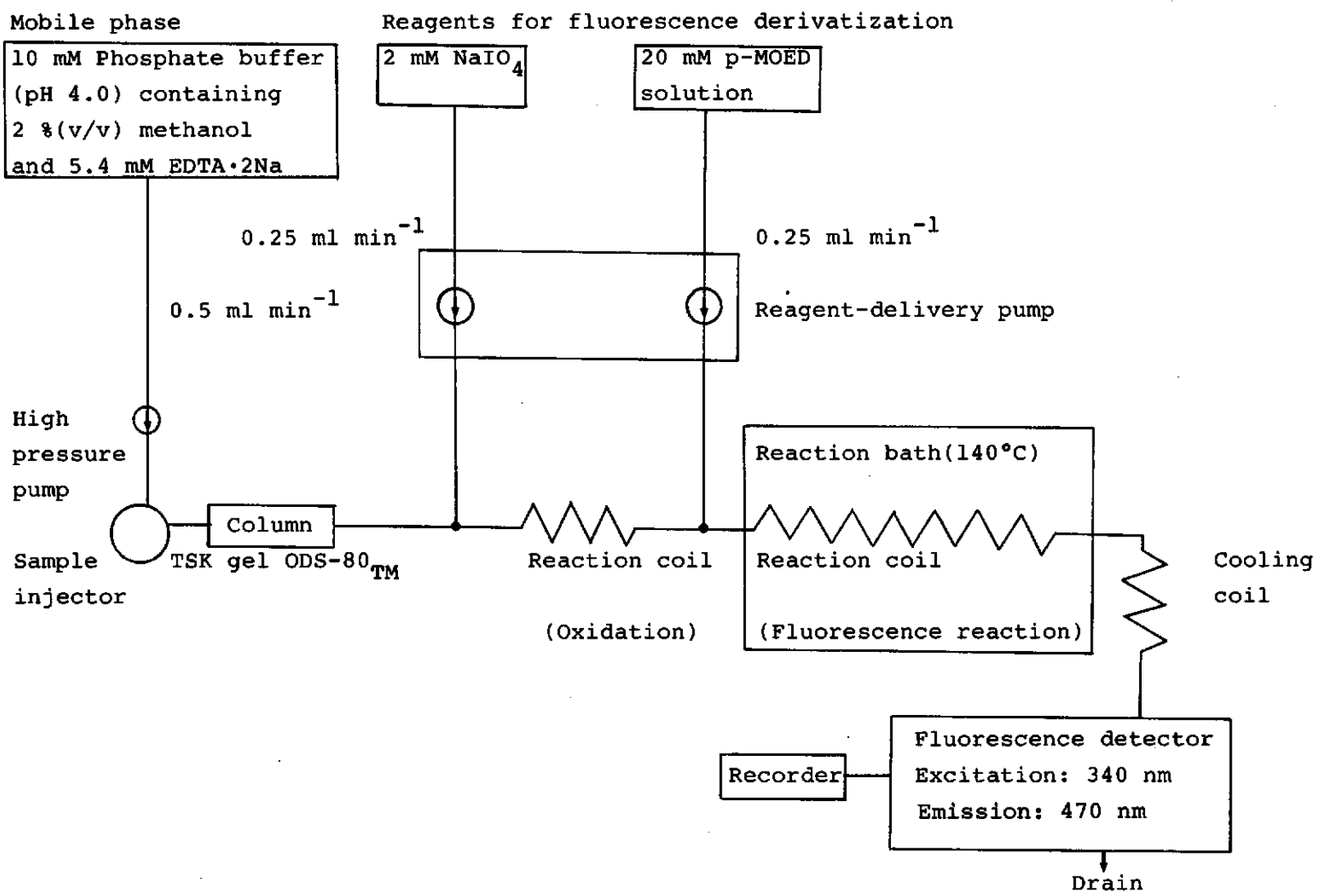

Fig. I Flow diagram of the HPLC system.

steel tube) placed in a Shimadzu (Kyoto, Japan) CRB$6 \mathrm{~A}$ reaction bath $\left(140^{\circ} \mathrm{C}\right)$ to develop fluorescence, and the reaction mixture was passed through an air-cooling coil ( $1 \mathrm{~m} \times 0.25 \mathrm{~mm}$ i.d., stainless-steel tube). The fluorescence intensity of the last effluent was monitored at $470-\mathrm{nm}$ emission with $340-\mathrm{nm}$ excitation (both spectral bandwidths were $15 \mathrm{~nm}$ ) using a Hitachi F1000 spectrofluorometer equipped with a flow cell $(12 \mu \mathrm{l})$. Peak heights were used for quantification.

\section{Sample preparation for HPLC}

Heparinized blood $(5 \mathrm{ml})$ was taken into a polyethylene tube and centrifuged at $1000 \mathrm{~g}$ for $15 \mathrm{~min}$ at $4^{\circ} \mathrm{C}$. The plasma and buffy coat were removed by aspiration. Subsequently, the erythrocytes were washed three times with $5 \mathrm{ml}$ of ice-cold physiological saline and the erythrocytes were immediately used for analysis.

To $300 \mu \mathrm{l}$ of the erythrocytes, $10 \mu \mathrm{l}$ of $5 \mu \mathrm{mol} \mathrm{ml}^{-1}$ XMP (internal standard) and $1 \mathrm{ml}$ of ice-cold $0.6 \mathrm{M}$ perchloric acid were successively added for deproteinization. The mixture was centrifuged at $1000 \mathrm{~g}$ for $10 \mathrm{~min}$. The supernatant $(300 \mu \mathrm{l})$ was mixed with $200 \mu \mathrm{l}$ of a $1 \mathrm{M} \mathrm{Na-K}$ phosphate buffer ( $\mathrm{pH} \mathrm{6.6)}$; the mixture was then briefly centrifuged. A $100 \mu \mathrm{l}$ aliquot of the supernatant was injected into the HPLC system. The sample preparation leading to acid-deproteinization after venipuncture must be carried out within $40 \mathrm{~min}$, since triphosphate ribonucleotides (such as ATP, inosine-5'-triphosphate (ITP) and guanosine-5'-triphosphate
(GTP)) decompose to the corresponding mono- and diphosphate ribonucleotides. ${ }^{13}$

\section{Results and Discussion}

\section{HPLC separation of ribonucleotides}

Nine ribonucleotides (ATP, ADP, adenosine-5'-monophosphate (AMP), GTP, guanosine-5'-diphosphate (GDP), guanosine-5'-monophosphate (GMP), uridine5'-diphosphate (UDP), inosine-5'-monophosphate (IMP) and XMP) were used as model compounds for HPLC separation. Three modes of HPLC separation (anionexchange ${ }^{5,6}$, reversed-phase and ion-pairing ${ }^{7}$ ) have been employed. An ion-exchange HPLC that used an anionexchanger column (TSK gel DEAE-2SW; Tosoh) required a highly concentrated buffer (approximately $1 \mathrm{M}$ phosphate buffer) for the separation and yielded broad peaks for the nine ribonucleotides. An ion-pair reversed-phase HPLC using a TSK gel ODS-80 column and tetrabutylammonium hydroxide as an ion-pair reagent in the mobile phase required a high flow rate of the mobile phase for the resolution of the ribonucleotides and it was unsuitable for the postcolumn derivatization reaction. Finally, it was found that the nucleotides could be satisfactorily separated on a reversed-phase column.(TSK gel ODS-80 TM) using an acidic phosphate buffer containing small amounts of methanol and EDTA.2 $\mathrm{Na}$ as a mobile phase. 


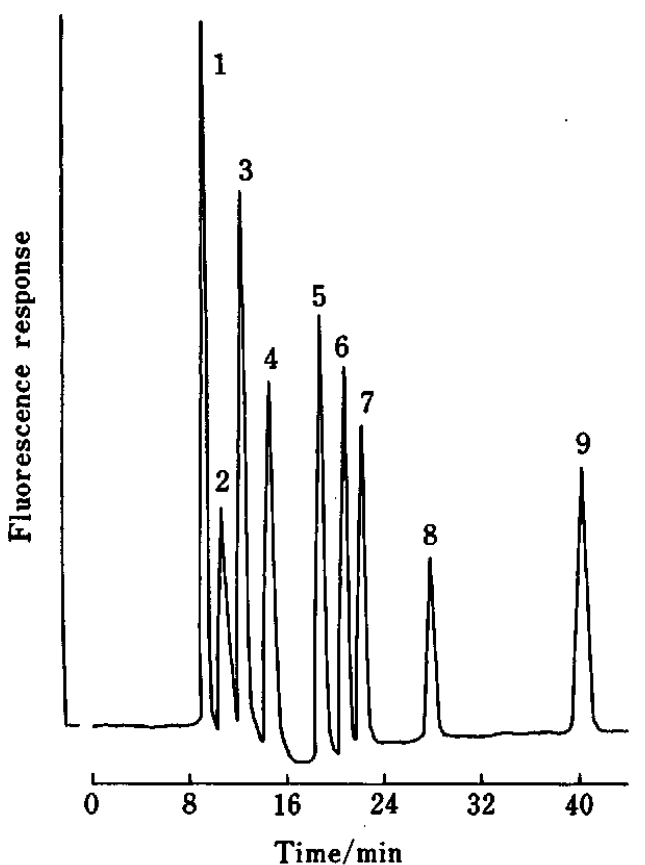

Fig. 2 Chromatogram of a standard mixture of nine ribonucleotides. Concentrations (nmol per $100-\mu 1$ injection volume): 1 nmol each. Peaks: 1, UDP; 2, GTP; 3, GDP; 4, ATP; 5, ADP; 6, GMP; 7, IMP; 8, XMP; 9, AMP.

Figure 2 shows a chromatogram obtained with a standard mixture of the nine ribonucleotides (retention times (min); 9.4 (UDP), 11.1 (GTP), 12.5 (GDP), 15.0 (ATP), 18.8 (ADP), 21.2 (GMP), 23.9 (IMP), 28.5 (XMP), 41.0 (AMP)). The eluates from all of the peaks have fluorescence excitation and emission maxima at $340 \mathrm{~nm}$ and $470 \mathrm{~nm}$, respectively; the fluorescence excitation and emission spectra were almost identical with those observed using a manual spectrofluorometric method. ${ }^{10}$

The retention times for the nucleotides decreased with increasing concentrations of sodium phosphate buffer and methanol in the mobile phase; the recommended concentrations $(10 \mathrm{mM}$ in sodium phosphate buffer and $2 \%(\mathrm{v} / \mathrm{v})$ in methanol) could afford a rapid and satisfactory separation. The retention times increased upon decreasing the $\mathrm{pH}$ of the phosphate buffer; the selected $\mathrm{pH}(4.0)$ gave complete separation. EDTA $2 \mathrm{Na}$ in the mobile phase served to sharpen the peaks for triphosphate ribonucleotides at a concentration of around $5.4 \mathrm{mM}$. EDTA $2 \mathrm{Na}$ may protect the HPLC column from adhesion of metal ions which cause the formation of a chelate complex with the triphosphate ribonucleotides.

\section{Conditions of postcolumn fluorescence derivatization}

A sodium periodate concentration ranging from 1 $3 \mathrm{mM}$ yielded the highest peaks for the nine nucleotides; $2 \mathrm{mM}$ was selected for reproducible results. Perchloric acid that was an acid required for the fluorescence reaction provided almost maximum peak heights in the concentration range $0.05-0.15 \mathrm{M}$ in a $p$-MOED solution; $0.14 \mathrm{M}$ was selected as being optimum. Water-miscible organic solvents (such as methanol, ethanol and acetonitrile) accelerated the fluorescence reaction. Of these solvents, ethanol was the most effective in a concentration range of $60-80 \%(v / v)$ in the $p$-MOED solution; $68 \%(\mathrm{v} / \mathrm{v})$ was employed. The highest peaks were attained at $p$-MOED concentrations of $15-25 \mathrm{M} ; 20 \mathrm{mM}$ was selected.

A $1-\mathrm{m}$ long oxidation reaction coil $(0.5 \mathrm{~mm}$ i.d. $)$ was most satisfactory. Fluorescence reaction coils $(0.5 \mathrm{~mm}$ i.d.) of 15-25 m gave almost maximum and constant peak heights; $20 \mathrm{~m}$ was used (the passage period of the reaction mixture was $3.9 \mathrm{~min}$ ). The peak heights increased with increasing the reaction temperature up to approximately $150^{\circ} \mathrm{C}$. A temperature higher than $150^{\circ} \mathrm{C}$ caused background noise in the chromatogram due to gas bubble formation in the reaction coil; $140^{\circ} \mathrm{C}$ was most acceptable.

The calibration graphs obtained by plotting the peak heights versus the amounts of the nine nucleotides $(0.1$ $10 \mathrm{nmol}$ per injection volume) were linear and passed through the origin. The detection limits (pmol in a injection volume of $100 \mu \mathrm{l} ; S / N=3$ ) were 14 (UDP), 38 (GTP), 19 (GDP), 25 (ATP), 24 (ADP), 29 (GMP), 38 (IMP), 67 (XMP) and 43 (AMP). The relative standard deviations $(n=10)$ for the nucleotides did not exceed $2.8 \%$.

\section{Determination of ribonucleotides in human erythrocytes}

Since XMP does not occur in human erythrocytes it was employed as an internal standard. Recoveries (mean \pm standard deviation $(\%), n=5)$ of the nucleotides ( $50 \mathrm{nmol}$ each) added to $300 \mu \mathrm{l}$ of erythrocytes in the recommended deproteinization procedure with perchloric acid were 92 \pm 1 (ATP), 67士2 (ADP), $85 \pm 2$ (IMP), $78 \pm 2$ (AMP) and $87 \pm 1$ (XMP). Two other deproteinization procedures that used acetonitrile and trichloroacetic acid, respectively, gave lower recoveries. Figure 3 shows a typical chromatogram obtained with human erythrocytes. The peak components were identified on the basis of the retention times in comparison with the standard solutions and by co-chromatography of both the standards and the samples. Peaks ascribable to ATP, GTP, ITP, GDP, inosine-5'-diphosphate (IDP), ADP, IMP and AMP were observed in the chromatogram. Although the late-eluting compounds (ATP, ADP, IMP and AMP) could be determined, the earlyeluting compounds (GTP, ITP, GDP and IDP) could not be quantified successfully because of insufficient resolution of the peaks. However, the resolution of the early-eluting compounds could be greatly improved by using the mobile phase lacking methanol; the lateeluting compounds were eluted much later with badly broadened peaks. Peak 10 in Fig. 3 appears to be due to a ribonucleotide, since the fluorescence excitation and emission maxima of the eluate were at 340 and $470 \mathrm{~nm}$, respectively. These are characteristic of ribonucleotides; the peak disappeared when $p$-MOED 


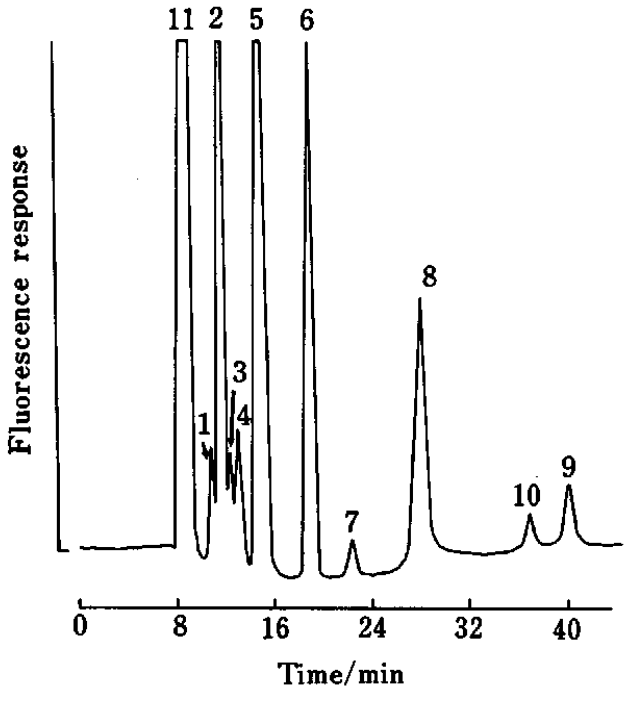

Fig. 3 Chromatogram obtained with erythrocytes from healthy person (male, 27 years old). Peaks and concentrations (nmol ml ${ }^{-1}$ ) in parentheses: 1, GTP; 2, ITP; 3, GDP; 4, IDP and/or others; 5, ATP (1007.4); 6, ADP (147.5); 7, IMP (7.5); 8, XMP (166.7, internal standard); 9, AMP (23.3); 10 , probably a ribonucleotide; 11 , unidentified.

was absent in the HPLC system.

Linear relationships were obtained between the ratios of the peak heights of ATP, ADP, IMP and AMP to that of XMP, and the amounts of ATP, ADP, IMP and AMP added in the range of $1-50 \mathrm{nmol}$ each to $300 \mu l$ of erythrocytes. The detection limits $(S / N=3)$ for ATP, ADP, IMP and AMP in erythrocytes were $1.8,1.7,2.8$ and $3.1 \mathrm{nmol} \mathrm{ml}^{-1}$ (corresponding to 25,24 , 38 and 43 pmol per injection volume), respectively. The relative standard deviations in repeated determinations $(n=5)$ of ATP, ADP, IMP and AMP were 2.1, 1.5, 1.9 and $2.3 \%$ at mean concentrations of $1007,148,7.5$ and $23.3 \mathrm{nmol} \mathrm{ml}^{-1}$ erythrocytes, respectively.

The concentrations of ATP, ADP, IMP and AMP in erythrocytes from healthy persons were determined by the present method (Table 1). The values agreed with data obtained by other workers. ${ }^{8,9,13,14}$

This method is fairly sensitive and highly selective. It should therefore be applicable to biomedical and bioanalytical investigations of ribonucleotides.

This work was partly supported by a Grant-in-Aid for Scientific Research from the Ministry of Education, Science and Culture, Japan.
Table 1 Concentrations of ribonucleotides in erythrocytes from healthy persons

\begin{tabular}{ccccl}
\hline \multirow{2}{*}{$\begin{array}{c}\text { Age } \\
\text { (year) }\end{array}$} & \multicolumn{4}{c}{ Concentration $/ \mathrm{nmol} \mathrm{m}^{-1}$} \\
\cline { 2 - 5 } ATP & ADP & IMP & AMP \\
\hline M38 & 1952 & 201 & 7.3 & 21.6 \\
M38 & 2144 & 189 & 3.2 & 15.6 \\
M32 & 1991 & 286 & 9.7 & 52.8 \\
M27 & 1984 & 229 & 8.5 & 27.2 \\
M26 & 2799 & 192 & 4.3 & 12.0 \\
M26 & 667 & 107 & 7.1 & 12.2 \\
M24 & 1007 & 147 & 7.5 & 23.3 \\
F24 & 1304 & 130 & 3.1 & 12.6 \\
M22 & 1309 & 216 & 10.9 & 32.4 \\
F21 & 1188 & 172 & 7.0 & 27.2 \\
& & & & \\
MeantSD & $1634 \pm$ & $187 \pm$ & $6.9 \pm$ & $23.7 \pm$ \\
& 609 & 49 & 2.5 & 11.9 \\
\hline
\end{tabular}

M, male; F, female.

\section{References}

1. M. H. Lerner and B. A. Lowy, J. Biol. Chem., 249, 959 (1974).

2. J. B. Prirchard, N. O'Conner, J. M. Oliver and R. D. Berlin, Am. J. Physiol, 229, 967 (1975).

3. C. H. de Verdier, A. Eriscon, F. Niklasson and T. Grotth, Acta Biol. Med. Germ., 40, 677 (1981).

4. C. T. Stannard and P. A. Gibbs, J. Biolumin. Chemilumin., 1, 3 (1986).

5. G. R. Bartlett, Biochim. Biophys. Acta, 156, 221 (1968).

6. A. Eriscon, F. Niklasson and C. H. de Verdier, Clin. Chim. Acta, 127, 47 (1983).

7. A. Werner, W. Siems, H. Schmidt, I. Rapoport, G. Gerber, R. T. Toguzov, Y. V. Tikhonov and A. M. Pimenov, J. Chromatogr., 421, 257 (1987).

8. M. Zakaria and P. R. Brown, J. Chromatogr., 226, 267 (1981).

9. P. Rotillan, A. Liras and P. Llorente, Anal. Biochem., 159, 377 (1986).

10. Y. Umegae, H. Nohta and Y. Ohkura, Chem. Pharm. Bull., 38, 452 (1990).

11. Y. Umegae, H. Nohta and Y. Ohkura, J. Chromatogr., in press (1990).

12. F. Vogtle and E. Goldschmitt, Chem. Ber., 109, 1 (1976).

13. B. M. Dean, D. Perrett and M. Sensi, Biochem. Biophys. Res. Commun., 80, 47 (1978).

14. W. R. A. Osborne, W. P. Hammond and D. C. Dale, J. Lab. Clin. Med., 105, 403 (1985).

(Received March 7, 1990) (Accepted April 8, 1990) 